\title{
Impaired Contractile Function and Mitochondrial Respiratory Capacity in Response to Oxygen Deprivation in a Rat Model of Pre-Diabetes
}

\author{
M. Faadiel Essop ${ }^{1^{*}}$, W.Y. Anna Chan ${ }^{2}$, Adamo Valle ${ }^{3}$, Francisco José García- \\ Palmer $^{3}$, Eugene F. Du Toit ${ }^{4}$
}

${ }^{1}$ Department of Physiological Sciences, Stellenbosch University, Stellenbosch, South Africa ${ }^{2}$ Hatter Heart Research Institute, Department of Medicine, University of Cape Town's Health Sciences Faculty, Cape Town, South Africa

${ }^{3}$ Grup de Metabolisme Energètic i Nutrició, Universitat de les Illes Balears, Palma de Mallorca, and Ciber Fisiopatología Obesidad y Nutrición (CB06/03) Instituto Salud Carlos III, Spain ${ }^{4}$ Department of Medical Physiology, Stellenbosch University Faculty of Health Sciences, Tygerberg, South Africa

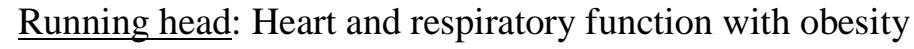

Word count: 4,611

*Corresponding author:

Dr. M. Faadiel Essop

Department of Physiological Sciences

Stellenbosch University

Mike De Vries Building

Merriman Avenue, Stellenbosch 7600

South Africa

Tel: $\quad+27218084507$

Fax: + 27218083145

E-mail: mfessop@sun.ac.za 


\section{Abstract}

Aim: Obesity is a major contributor to the global burden of disease and is closely associated with the development of type 2 diabetes and cardiovascular diseases. This study tested the hypothesis that mitochondrial respiratory capacity of the pre-diabetic heart is decreased leading to impaired contractile function and tolerance to ischaemia/reperfusion.

Methods: Eight-week-old male Wistar rats were fed a high caloric diet for 16 weeks whereafter anthropometric, metabolic, cardiac and mitochondrial parameters were evaluated versus agematched lean controls. Cardiac function (working heart perfusions) and mitochondrial respiratory capacity were assessed at baseline and in response to acute oxygen deprivation.

Results: Rats fed the high caloric diet exhibited increased body weight and visceral fat versus the control group. Heart weights of obese rats were also increased. Triglyceride, fasting plasma insulin and free fatty acid levels were elevated, while HDL cholesterol levels were reduced in the obese group. Contractile function was attenuated at baseline and further decreased after subjecting hearts to ischemia-reperfusion. Myocardial infarct sizes were increased while ADP phosphorylation rates were diminished in obese rats. However, no differences were found for mtDNA levels and the degree of oxidative stress-induced damage.

Conclusions: These data show that decreased mitochondrial bioenergetic capacity in pre-diabetic rat hearts may impair respiratory capacity and reduce basal contractile function and tolerance to acute oxygen deprivation.

Keywords: Metabolic syndrome; obesity; myocardial infarction; mitochondrial respiration 


\section{Introduction}

The prevalence of obesity is reaching global epidemic proportions and it is a major contributor to increased prevalence of the metabolic syndrome and type 2 diabetes (Eckel et al. 2005, Kelly et al. 2008). Insulin resistance and type 2 diabetes is characterized by metabolic derangements that may contribute to cardiac contractile dysfunction in the absence of atherosclerosis and hypertension, i.e. the diabetic cardiomyopathy (reviewed by Boudina \& Abel 2007a). For example, the diabetic heart displays higher rates of fatty acid $\beta$-oxidation (FAO) and a corresponding reduction of carbohydrate utilization (Carley \& Severson 2005). Furthermore, previous studies demonstrated that increased myocardial oxygen consumption and reduced cardiac efficiency (work/myocardial oxygen consumption) may be important factors contributing to contractile dysfunction of the diabetic heart (How et al. 2006, Boudina \& Abel 2006).

The precise mechanisms underlying this process are unclear although fatty acid-induced mitochondrial uncoupling and lowered mitochondrial ATP production have been implicated (Boudina et al. 2005). Moreover, increased mitochondrial reactive oxygen species (ROS) production by diabetic hearts is proposed to contribute to uncoupling of mitochondrial oxidative phosphorylation and oxygen wastage (Boudina et al. 2007b). However, most studies investigating metabolic perturbations and its effects on cardiac function were performed using experimental models of overt type 2 diabetes. Furthermore, mitochondrial studies were largely performed on genetic mouse models of obesity and insulin resistance (Boudina et al. 2005, Boudina et al. 2007b). Since mutations in the leptin gene or its receptor are rare in humans and leptin may have direct effects on cardiac function, it would be useful to assess the effects modern-day eating habits (high caloric diet) and obesity on a normal genetic background on mitochondrial energetics as emphasized in a recent review article by Abel et al. (2008). Importantly, it will be of great benefit to evaluate whether these changes occur at an earlier stage during disease progression, i.e. in the pre-diabetic state compared to more advanced stages of disease progression as studied before. In light of this, we employed a rat model of obesity- 
induced pre-diabetes to test the hypothesis that mitochondrial bioenergetic capacity of the prediabetic heart is decreased leading to impaired contractile function.

\section{Materials and Methods}

Animals

8-week-old male Wistar rats were housed at $22.0 \pm 0.5^{\circ} \mathrm{C}$ on a 12 -h light/dark cycle and divided into 2 groups, i.e. fed standard chow (60\% carbohydrate, 30\% protein, $10 \%$ fat) versus a high caloric diet (65\% carbohydrate, 19\% protein, 16\% fat) as described before (Du Toit et al. 2008). After a 16-week feeding period, rats were weighed and thereafter anesthetized using intraperitoneal pentobarbitone sodium (12 mg/kg). Hearts were rapidly excised and weighed to determine heart/body weight ratios. All animals were treated in accordance with the Principles of Laboratory Animal Care of the South African Medical Research Council and the Guide for the Care and use of Laboratory Animals of the National Academy of Sciences (NIH publication No. 85-23, revised 1996). The study was also ethically approved by the Committee for Experimental Animal Research of the Faculty of Health Sciences (Tygerberg campus of Stellenbosch University, South Africa).

\section{Metabolic assays}

During excision of the heart, blood samples were collected in EDTA tubes for glycosylated haemoglobin (HbA1c) and free fatty acid (FFA) measurements; potassium oxalate/sodium fluoride tubes for glucose determinations; and serum separation tubes for lipid analysis. Where appropriate, samples were centrifuged at $1500 \mathrm{~g}$ for $10 \mathrm{~min}$ at $4^{\circ} \mathrm{C}$ and plasma/serum stored at -

$20^{\circ} \mathrm{C}$ for later use. However, blood glucose and HbA1c measurements were performed on the day of sample collection. 


\section{Serum assays}

Serum total cholesterol (TC), high density lipoprotein (HDL)-cholesterol and triglycerides were measured using enzymatic and colorimetric methods as described previously (Esterhuyse et al. 2005). Plasma FFA levels were determined using an enzymatic colorimetric assay (Roche, Penzberg, Germany). Serum insulin levels were measured using a radio-immunoassay-based kit (Diagnostic Products Corporation, Los Angeles, CA). Blood glucose levels were measured using the glucose oxidase method with one-touch test strips (Accu-Check, Roche Diagnostics, Mannheim, Germany). Blood HbA1c levels were determined using the glycosal spectrophotometric method (National Health Laboratory Services, Tygerberg Hospital, Cape Town, South Africa).

\section{Blood pressure measurements}

After 16 weeks, systolic blood pressure was determined using the standard tail-cuff method (Bunag 1973). Briefly, rats were sedated with thiopentone sodium (20 mg/kg) while a tail-cuff and a pneumatic pulse sensor were fitted. The tail-cuff was linked to a sphygmomanometer and the pulse sensor to a Lectromed Multitrace 2 chart recorder (Rue Fondon, Channel Islands). The tail-cuff was gradually inflated until the pulse pressure curve on the chart recorder disappeared. The sphygmomanometer linked to the tail cuff was then deflated until the pulse pressure curve reappeared on the chart recorder. The sphygmomanometer pressure that coincided with the reappearance of the pulse pressure curve was considered the systolic blood pressure. The procedure was repeated three times and the mean systolic pressure was determined.

\section{Isolated heart perfusions}

After 16 weeks feeding, animals were anesthetized using intraperitoneal pentobarbitone sodium (12 mg/kg), hearts rapidly excised and placed in ice-cold Krebs-Henseleit perfusion buffer (118 
$\mathrm{mmol} / \mathrm{L} \mathrm{NaCl}, 4.7 \mathrm{mmol} / \mathrm{L} \mathrm{KCl}, 1.2 \mathrm{mmol} / \mathrm{L} \mathrm{MgSO}_{4} .7 \mathrm{H}_{2} \mathrm{O}, 1.25 \mathrm{mmol} / \mathrm{L} \mathrm{CaCl}, 25 \mathrm{mM} \mathrm{NaHCO}$, $1.2 \mathrm{mmol} / \mathrm{L} \mathrm{KH}_{2} \mathrm{PO}_{4}$ and $10 \mathrm{mM}$ glucose). Hearts were subsequently transferred to a working heart perfusion apparatus and perfused with Krebs-Henseleit buffer equilibrated with $95 \% \mathrm{O}_{2} /$ $5 \% \mathrm{CO}_{2}$ at $37^{\circ} \mathrm{C}$ and $100 \mathrm{~cm} \mathrm{H}_{2} \mathrm{O}$ pressure. Retrograde perfusion was initiated within $45 \mathrm{sec}$ of excision of the heart.

\section{Functional recovery and myocardial infarct size measurements}

Hearts were initially perfused in the Langendorff mode for a 10 min stabilization period, thereafter for 20 min in the working heart mode. Pre-ischemic aortic output (AO), coronary flow (CF) and aortic systolic and diastolic pressure (ADP and ASP) was determined before hearts were subjected to 15 min normothermic global ischemia $\left(37^{\circ} \mathrm{C}\right)$. Subsequently, hearts were reperfused in the Langendorff mode for 5 min before switching to the working heart mode for an additional 20 min. Reperfusion functional parameters were again documented at 10, 15 and 20 minutes, respectively. Percentage aortic output recovery was determined as follows: Aortic output recovery $=(\mathrm{AO}$ at reperfusion $[20 \mathrm{~min}] / \mathrm{AO}$ at pre-ischemia $[20 \mathrm{~min}]) \times 100$.

In a separate series of hearts myocardial infarct size was determined as described previously (Du Toit et al. 2008). Briefly, hearts were perfused as above before being subjected to 40 min regional ischemia at $37^{\circ} \mathrm{C}$. Regional ischemia was induced by ligation of the left anterior descending coronary artery using a silk suture. The suture was then released and the heart reperfused for 30 mins before the coronary artery was re-occluded. After re-occlusion, a solution of $2.5 \%$ Evans blue was injected into the coronary arteries to delineate the area at risk. Hearts were then frozen, cut into slices and incubated in sodium phosphate buffer containing 1\% w/v triphenyltetrazolium chloride (TTC) for 15 min to visualize the unstained infarcted region. Infarct and risk zone areas were determined with planimetry and infarct area was expressed as a percentage of the area at risk (Du Toit et al. 2008). 


\section{Mitochondrial isolation and measurement of respiratory function}

For a separate set of rats (non-perfused), mitochondria were isolated as described before, with modifications (Essop et al. 2007). In brief, ventricular tissues were minced and gently homogenized in $3 \mathrm{ml}$ ice-cold potassium-EDTA isolation buffer (0.18 M KCL, $10 \mathrm{mM}$ EDTA) using a glass homogenizer (Tenbroek, Netherlands). After differential centrifugation the mitochondrial pellet was gently resuspended in $100 \mu \mathrm{l}$ of incubation buffer $(10 \mathrm{mM}$ Tris- $\mathrm{HCl}$, $250 \mathrm{mM}$ sucrose, $8.5 \mathrm{mM} \mathrm{KH}_{2} \mathrm{PO}_{4}$ ). It is likely that we isolated subsarcolemmal mitochondria as released by mechanical disruption, unlike interfibrillar mitochondrial isolation that requires protease treatment and additional centrifugation steps (Palmer et al. 1977) that were not performed in this study. Mitochondrial protein concentrations were determined and respiratory capacity was measured polarographically at $25^{\circ} \mathrm{C}$ as described (Essop et al. 2007). Two sets of experiments were performed, i.e. a) $12.5 \mathrm{mM}$ glutamate or b) $54 \mu \mathrm{M}$ palmitoyl-L-carnitine and 3.3 mM malate used as oxidative substrates. Analyses were only performed when the respiratory control ratio (RCR), i.e. the ratio of state 3 respiration: state 4 respiration, was $\geq 3$.

\section{Mitochondrial respiratory function in response to anoxic stress}

To test the ability of mitochondria to withstand oxidative stress, mitochondria (still incubated with respective oxidative substrates) were exposed to 20 min of oxygen lack followed by 6 min of reoxygenation as described (Essop et al. 2007). Recovery of respiratory function was evaluated by determining the ratio of post-anoxia state 3 respiration over state 3 respiration prior to oxygen deficiency. All mitochondrial polarographic studies were normalized to total mitochondrial protein. 


\section{Extraction and quantification of mitochondrial DNA}

Mitochondrial DNA (mtDNA) was extracted by digestion with proteinase $\mathrm{K}(100 \mu \mathrm{g} / \mu \mathrm{l})$ in a buffer containing $50 \mathrm{mM} \mathrm{KCl,} 10 \mathrm{mM}$ Tris-HCl, $2.5 \mathrm{mM} \mathrm{MgCl}_{2}$ and $0.5 \%$ Tween 20 . Homogenate samples were incubated overnight at $37^{\circ} \mathrm{C}$ and then boiled for $5 \mathrm{~min}$ in order to inactivate the enzyme. Mitochondrial DNA was linearized by digestion with BclI restriction enzyme for $3 \mathrm{~h}$ at $50^{\circ} \mathrm{C}$ and boiled for $5 \mathrm{~min}$. Samples were centrifuged at 7,000 $\mathrm{g}$ for $5 \mathrm{~min}$ and the resulting supernatant was used for amplification. A quantitative PCR assay was adapted to for light cycler amplification (Koekemoer et al. 1998). PCR was performed to amplify a 162-nts fragment of the mitochondrial NADH dehydrogenase subunit 4 gene. The primer sequences were 5'-TACACGATGAGGCAACCAAA-3' and 5'-GGTAGGGGGTGTGTTGTGAG-3'. Increasing amounts of template were amplified in parallel reactions to obtain a standard curve. Amplification was carried out in a LightCycler rapid thermal cycler system (Roche, Switzerland) using a total volume of $10 \mu \mathrm{l}$ containing $0.375 \mu \mathrm{M}$ of each primer, $3 \mathrm{mM} \mathrm{MgCl}_{2}, 1 \mu \mathrm{l}$ LightCycler-FastStart DNA Master SYBR Green I (Roche, Switzerland), and $2.5 \mu \mathrm{l}$ of sample prepared as described above. After initial denaturation $\left(95^{\circ} \mathrm{C}, 10 \mathrm{~min}\right)$, PCR reactions were cycled 35 times with the following parameters: denaturation at $95^{\circ} \mathrm{C}$ for $10 \mathrm{~s}$, annealing at $60^{\circ} \mathrm{C}$ for $12 \mathrm{~s}$, extension at $72^{\circ} \mathrm{C}$ for $12 \mathrm{~s}$. Total DNA was measured using the diaminobenzoic acid method for mtDNA correction (Thomas \& Farquhar 1978).

\section{Measurement of thiobarbituric acid-reactive substances}

Lipid peroxidation levels or thiobarbituric acid-reactive substances (TBARS) were determined as malondialdehyde-thiobarbituric acid adducts as described (Buege \& Aust 1978). Peroxidation levels were measured spectrophotometrically at $532 \mathrm{~nm}$, using a molar extinction coefficient of $1.56 \times 10^{5} \mathrm{M}^{-1} / \mathrm{cm}$, and expressed as nanomoles of TBARS per milligram of protein. 


\section{Measurement of carbonyl content}

Carbonyl groups were quantified using the Oxyblot protein oxidation detection kit (Chemicon, Chandlers Ford, UK). Here 2,4-dinitrophenyhydrazine (DNPH) derivatization was carried out for $15 \mathrm{~min}$ on $5 \mu \mathrm{g}$ of homogenate protein following the manufacturer's instructions. Proteins were transferred to nitrocellulose filters by means of a slot-blot system (Bio-Rad, Hercules, CA). After incubation with anti-DNP antibody, blots were developed using the ECL detection system on ChemiDoc XRS (Bio-Rad, Hercules, CA). Bands were quantified using the Quantity One software package. To determine specificity, the oxidized proteins provided by the kit were included as a positive control. Treatment of samples with a control solution served as a negative control for the DNPH derivatization.

\section{Statistics}

All data are expressed as mean \pm SEM. The unpaired Students t-test was used to determine statistical significance. For multiple comparisons significance was determined by ANOVA followed by the Bonferroni test. $\mathrm{P}<0.05$ was considered statistically significant.

\section{Results}

Body weight (BW) was significantly higher in rats fed the high caloric diet compared to controls (Table 1). The obese group also displayed increased heart weight to body weight (HW: BW) and left ventricle to body weight ratios (LV: BW). Moreover, triglycerides, fasting plasma insulin, HbA1c and FFA levels were elevated, while HDL cholesterol levels were reduced in the obese group versus controls (Table 1). However, fasting plasma glucose levels and systolic blood pressure did not significantly differ between control and obese rats, while visceral fat was higher after the suboptimal feeding period ( $<<0.05$ vs. matched controls). Importantly, HbA1c levels were not above $6.5 \%$ value in the high caloric diet fed rats. Values below this limit is indicative of good blood glucose management in these animals. 
We next assessed ex vivo contractile functional parameters and myocardial infarct size after ischemia. Here the pre-ischaemic baseline aortic output was reduced in the obese group (41.0 \pm 1.2 vs. $34.0 \pm 1.2 \mathrm{ml} / \mathrm{min})$ ( $<<0.05$ vs. controls) (Fig. 1a). Furthermore, the aortic output recovery was $\sim 25-30 \%$ lower in the obese group after ischemia-reperfusion (Fig.1b). For infarct size studies, the area of the left ventricle at risk was similar for both groups, i.e. $48.7 \pm 1.6 \%$ of the left ventricular volume for controls and $50.4 \pm 1.7 \%$ for obese rats. In agreement with the functional data, myocardial infarct size after ischemia/reperfusion was increased in the obese group (24.3 \pm 2.1 vs. $42.9 \pm 1.8 \%$ of area at risk) ( $<<0.0001$ vs. controls) (Fig. 1c).

We employed two different mitochondrial oxidative substrates to gain insight regarding fuel substrate utilization. State 2 respiration was decreased $(22.2 \pm 1.0$ vs. $19.2 \pm 0.6 \mathrm{nmol} / \mathrm{min} / \mathrm{mg}$ protein) in the obese group when malate palmitoyl-L-carnitine (MPC) was used as oxidative substrate ( $<0.05$ vs. controls) (Table 2 ). Lower state 2 respiration in the obese rats merely reflects low endogenous ADP levels of which the origin is unclear. State 3 respiration and ADP/O ratios were similar for obese and control mitochondria (using both oxidative substrates). State 4 respiration did not differ when MPC was employed as oxidative substrate, but was lower for the obese group when glutamate was used as substrate. We also found that the ADP phosphorylation rate was decreased in the obese group $(278.1 \pm 8.3$ vs. $245.6 \pm 11.3 \mathrm{nmol}$ $\mathrm{ADP} / \mathrm{min} / \mathrm{mg}$ protein) ( $<<0.05$ vs. controls) when MPC was employed as oxidative substrate.

To further assess the effects of diet-induced obesity on mitochondrial respiratory function, we exposed mitochondria to 15 minutes oxygen lack followed by 6 minutes of reoxygenation. We subsequently determined state 3 respiration (using MPC as substrate) as an index of mitochondrial function in response to the anoxic stress. Here, the percentage recovery of state 3 mitochondrial respiration was diminished in mitochondria isolated from the obese group (51.7 \pm 8.2 vs. $28.7 \pm 5.4 \mathrm{nmol} \mathrm{O}_{2} / \mathrm{min} / \mathrm{mg}$ protein) ( $<<0.05$ vs. controls) (Fig. 2).

We also investigated potential mechanisms to explain decreased contractile and mitochondrial respiratory function in obese rats. Here we determined mtDNA levels but found no 
significant differences between the obese and control groups, i.e. $100 \pm 10.8$ vs. $133 \pm 29.5$ mtDNA/ng tissue. We also evaluated the effects of oxidative stress and found no significant differences for the degree of protein carbonylation $\left(100 \pm 16\right.$ vs. $\left.133 \pm 25 \mathrm{int} / \mathrm{mm}^{2}\right)$ and lipid peroxidation (TBARS; $6.6 \pm 1$ vs. $5.6 \pm 0.3$ nmoles/mg protein) for control versus obese rats.

\section{Discussion}

Obesity is a major contributor to the global burden of disease and is closely associated with the development of type 2 diabetes and cardiovascular diseases. In light of this, we investigated cardiac contractile function and mitochondrial respiratory capacity in a rat model of obesityinduced pre-diabetes. The main finding of this study is that pre-diabetic obese rats displayed reduced baseline cardiac contractile function and mitochondrial bioenergetic capacity, and impaired contractile function and decreased mitochondrial respiratory capacity in response to acute oxygen lack.

Our rodent model of diet-induced obesity closely resembles the pre-diabetic state/ metabolic syndrome that are typically characterized by insulin resistance, dyslipidemia (high triglycerides, low HDL cholesterol levels), hypertension and visceral obesity (Miranda et al. 2005). Here we found that obese rats had increased visceral fat, dyslipidemia and higher plasma insulin levels. Since elevated circulating FFA levels are causally linked to the onset of insulin resistance (Delarue \& Magnan 2007) and we previously reported a higher homeostasis model assessment (HOMA) index for obese rats using the same model (Du Toit et al. 2008), our data

strongly support an insulin resistant state. Moreover, when the feeding regimen is extended to the 30-week time point, we observe that fasting insulin and glucose levels continue to increase versus 16-week-old rats characterized in this study (Essop et al., unpublished data). In parallel, the HOMA index is further increased at the 30 -week time point, indicating progressive loss of insulin sensitivity. 
Obese rats also exhibited a degree of cardiac remodeling, i.e. the development of cardiac hypertrophy. These data are consistent with previous studies reporting a hypertrophic response in obese/type 2 diabetic rodents (Yue et al. 2007, Du Toit et al. 2008). The initial hypertrophic response is usually characterized by increased wall thickness followed by progressive dilatation and contractile dysfunction. We found that contractile function of obese rats was lower at baseline and further decreased after subjecting hearts to ischemia-reperfusion. In parallel, myocardial infarct sizes were increased. We found that infarct sizes remain more or less the same when different substrates (e.g. glucose, palmitate, insulin) are employed for perfusion studies (Essop et al., unpublished data). Glutamate and malate-palmitoyl-L-carnitine are not substrates commonly used in isolated heart perfusions and more suitable for isolated mitochondrial studies. These findings are in agreement with epidemiological data showing that the metabolic syndrome is associated with a significantly greater risk of cardiovascular disease (Wilson et al. 2005). However, not many laboratory studies have assessed cardiac damage in response to ischemia-reperfusion in the pre-diabetic state. Multiple factors may play a role in this process, e.g. altered lipoprotein levels, ectopic cardiac triglyceride lipid deposition, activation of inflammatory pathways, metabolic derangements and perturbed mitochondrial respiratory capacity (reviewed by Boudina \& Abel 2006).

In line with our hypothesis, we next evaluated whether altered mitochondrial respiratory capacity may provide a potential mechanism for attenuated cardiac function in obese rats. Overall, we did not find many differences between control and pre-diabetic rats. However, ADP phosphorylation rates were diminished in obese rats when a fatty acid was employed as a fuel substrate. Moreover, we found that state 4 respiration (malate-palmitoyl-L-carnitine as substrate) was not significantly altered in the obese group, suggesting no significant degree of mitochondrial uncoupling. Although state 4 respiration was lower for the obese group when glutamate was used as oxidative substrate, we are of the opinion that malate-palmitoyl-carnitine is a more representative substrate of uncoupling in the heart since fatty acids are the major fuel 
substrate of the adult mammalian heart. Moreover, state 4 respiration has limitations as a marker for proton leak since additional, '’non-uncoupling', factors e.g. substrate oxidation and efficiency of electron transport may influence this parameter.

To gain further insight into possible mitochondrial respiratory defects, we exposed isolated heart mitochondria to an acute anoxic insult followed by reperfusion and found that polarographic mitochondrial oxygen consumption was impaired for obese rats. Our mitochondrial data therefore suggest that attenuated mitochondrial bioenergetic capacity may contribute to the decreased respiratory capacity observed in response to acute oxygen lack.

We also tested the degree of oxidative damage since increased fatty acid oxidation rates are linked to elevated levels of ROS production, thereby contributing to lipid and protein peroxidation (Boudina et al. 2007). However, we found no significant degree of oxidative stressinduced damage. Moreover, since mtDNA levels were also not significantly altered our data therefore support a pre-diabetic state, i.e. with a limited degree of oxidative stress and damage to intracellular structural components. We are unclear regarding the precise mechanisms underlying decreased mitochondrial bioenergetic capacity in obese rats. However, we propose that downregulation of several nuclear-encoded mitochondrial genes and/or reduced protein levels of mitochondrial respiratory chain complexes with type 2 diabetes animal models (Boudina et al. 2005) may be contributing factors to this process. Alternatively, disorganization of mitochondrial ultrastructure may also be implicated (Dong et al. 2006). These possibilities require further investigation.

In summary, we have established a rodent model of pre-diabetes resembling the human metabolic syndrome. Here obese rats displayed increased myocardial damage and attenuated respiratory capacity in response to acute oxygen deprivation. We propose that decreased mitochondrial bioenergetic capacity may play an important role in this process. Investigations to 
enhance this process may therefore result in novel therapeutic interventions to limit the onset of the diabetic cardiomyopathy.

\section{Acknowledgements}

The authors wish to thank Mr. Wayne Smith for technical assistance. This work was supported by South African Medical Research Council and the South African National Research Foundation (to M.F. Essop), and the South African National Research Foundation (to E. F. Du Toit).

\section{Conflict of interest}

None. 


\section{References}

Abel, E.D., Litwin, S.E. \& Sweeney, G. 2008. Cardiac remodeling in obesity. Physiol Rev 88, 389-419.

Boudina, S., Sena, S., O'Neill, B.T., Tathireddy, P., Young, M.E. \& Abel, E.D. 2005. Reduced mitochondrial oxidative capacity and increased mitochondrial uncoupling impair myocardial energetics in obesity. Circulation 112, 2686-2695.

Boudina, S. \& Abel, E.D. 2006. Mitochondrial uncoupling: a key contributor to reduced cardiac efficiency in diabetes. Physiology 21, 250-258.

Boudina, S. \& Abel, E.D. 2007a. Diabetic cardiomyopathy revisited. Circulation 115, 32133223.

Boudina, S., Sena, S., Theobald, H., Sheng, X., Wright, J.J., Hu, X.X., Aziz, S., Johnson, J.I., Bugger, H., Zaha, V.G. \& Abel, E.D. 2007b. Mitochondrial energetics in the heart in obesityrelated diabetes: direct evidence for increased uncoupled respiration and activation of uncoupling proteins. Diabetes 56, 2457-2466.

Buege, J.A. \& Aust, S.D. 1978. Microsomal lipid peroxidation. Methods Enzymol 52, 302-310.

Bunag, R.D. 1973. Validation in awake rats of a tail-cuff method for measuring systolic pressure. J Appl Physiol 34, 279-282. 
Carley, A.N. \& Severson, D.L. 2005. Fatty acid metabolism is enhanced in type 2 diabetic hearts. Biochim Biophys Acta 1734, 112-126.

Delarue, J. \& Magnan, C. 2007. Free fatty acids and insulin resistance. Curr Opin Clin Nutr Metab Care 10, 142-148.

Dong, F., Zhang, X., Yang, X., Esberg, L.B., Yang, H., Zhang, Z., Culver, B. \& Ren, J. 2006. Impaired cardiac contractile function in ventricular myocytes from leptin-deficient ob/ob obese mice. J Endocrinol 188, 25-36.

Du Toit, E.F., Smith, W., Muller, C., Strijdom, H., Stouthammer, B., Woodiwiss, A.J., Norton, G.R. \& Lochner, A. 2008. Myocardial susceptibility to ischemic-reperfusion injury in a prediabetic model of dietary-induced obesity. Am J Physiol Heart Circ Physiol 294, H23362343.

Eckel, R.H., Grundy, S.M. \& Zimmet, P.Z. 2005. The metabolic syndrome. Lancet 365,14151428.

Essop, M.F., Chan, W.Y. \& Taegtmeyer, H. 2007. Metabolic gene switching in the murine female heart parallels enhanced mitochondrial respiratory function in response to oxidative stress. FEBS J 274, 5278-5284.

Esterhuyse, A.J., du Toit, E.F., Benadè, A.J. \& van Rooyen, J. 2005. Dietary red palm oil improves reperfusion cardiac function in the isolated perfused rat heart of animals fed a high cholesterol diet. Prostaglandins Leukot Essent Fatty Acids 72, 153-161. 
How, O.J., Aasum, E., Severson, D.L., Chan, W.Y., Essop, M.F. \& Larsen, T.S. 2006. Increased myocardial oxygen consumption reduces cardiac efficiency in diabetic mice. Diabetes 55, 466-473.

Kelly, T., Yang, W., Chen, C.S., Reynolds, K. \& He, J. 2008. Global burden of obesity in 2005 and projections to 2030. Int J Obes 32, 1431-1437.

Koekemoer, T.C., Downing, T.G. \& Oelofsen, W. 1998. An alternative PCR assay for quantifying mitochondrial DNA in crude preparations. Nucleic Acids Res 26, 2829-2830.

Miranda, P.J., DeFronzo, R.A., Califf, R.M. \& Guyton, J.R. 2005. Metabolic syndrome: definition, pathophysiology, and mechanisms. Am Heart J 149, 33-45.

Palmer, J.W., Tandler, B. \& Hoppel, C.L. 1977. Biochemical properties of subsarcolemmal and interfibrillar mitochondria isolated from rat cardiac muscle. J Biol Chem 252, 8731-8739.

Thomas, P. S., Farquhar, M. N. 1978. Specific measurement of DNA in nuclei and nucleic acids using diaminobenzoic acid. Anal Biochem 89, 35-44.

Wilson, P.W., D'Agostino, R.B., Parise, H., Sullivan, L. \& Meigs, J.B. 2005. Metabolic syndrome as a precursor of cardiovascular disease and type 2 diabetes mellitus. Circulation 112, 3066-3072.

Yue, P., Arai, T., Terashima, M., Sheikh, A.Y., Cao, F., Charo, D., Hoyt, G., Robbins, R.C., Ashley, E.A., Wu, J., Yang, P.C. \& Tsao, P.S. 2007. Magnetic resonance imaging of progressive cardiomyopathic changes in the db/db mouse. Am J Physiol Heart Circ Physiol 292, H2106-2118. 
Table 1 Baseline characterization of obese and control rats

\begin{tabular}{llc}
\hline & Control & Obese \\
Body weight (BW) (g) & $507.7 \pm 7.4$ & $588.6 \pm 8.1^{\# \#}$ \\
Heart weight (HW) (g) & $1.3 \pm 0.02$ & $1.5 \pm 0.04^{\# \#}$ \\
HW:BW ratio & $2.4 \pm 0.1$ & $2.6 \pm 0.1^{*}$ \\
LV:BW ratio & $2.4 \pm 0.1$ & $2.6 \pm 0.1^{*}$ \\
Total cholesterol (mmol/L) & $1.4 \pm 0.1$ & $1.3 \pm 0.1$ \\
HDL cholesterol (mmol/L) & $1 \pm 0.02$ & $0.6 \pm 0.03^{\#}$ \\
Triglycerides (mmol/L) & $0.7 \pm 0.1$ & $1.9 \pm 0.2^{\#}$ \\
FFA (mmol/L) & $0.8 \pm 0.1$ & $1.7 \pm 0.3^{*}$ \\
Glucose (fasting) (mmol/L) & $4.8 \pm 0.4$ & $5.3 \pm 0.2$ \\
Insulin (fasting) ( $\boldsymbol{H I U} / \mathbf{m L})$ & $27.8 \pm 3.5$ & $43.4 \pm 4.2^{*}$ \\
HbA1c (\%) & $3.5 \pm 0.1$ & $4 \pm 0.1^{* *}$ \\
SBP (mmHg) & $148 . \pm 3.9$ & $159 . \pm 3.9$ \\
Visceral fat (\%) & $29.8 \pm 1.6$ & $49.5 \pm 2.2^{*}$ \\
\hline
\end{tabular}

8 week-old male Wistar rats were fed a high caloric diet for 16 weeks and compared to matched controls on a standard laboratory chow. Values are expressed as mean \pm SEM ( $\underline{\mathbb{q} 5}$ animals). * $\mathrm{p}<0.05 ; * * \mathrm{p}<0.01,{ }^{*} \mathrm{p}<0.001$ and ${ }^{\# \#} \mathrm{p}<0.0001$ compared to control rats. 
Table 2 Cardiac mitochondrial respiratory function of obese and control rats

\begin{tabular}{|c|c|c|c|c|}
\hline & \multicolumn{2}{|c|}{ Glutamate } & \multicolumn{2}{|c|}{$\begin{array}{l}\text { Malate and palmitoyl-L- } \\
\text { carnitine (MPC) }\end{array}$} \\
\hline & Control & Obese & Control & Obese \\
\hline $\begin{array}{c}\text { State } 2 \text { respiration } \\
\text { (nmol/min/mg protein) }\end{array}$ & $11.2 \pm 1.1$ & $10.8 \pm 1.2$ & $22.2 \pm 1$ & $19.2 \pm 0.6^{*}$ \\
\hline $\begin{array}{l}\text { State } 3 \text { respiration (nmol } \\
\qquad \mathrm{O}_{2} / \mathrm{min} / \mathrm{mg} \text { protein) }\end{array}$ & $123.4 \pm 15.9$ & $121.5 \pm 8.6$ & $126.2 \pm 4.6$ & $117.1 \pm 5$ \\
\hline $\begin{array}{c}\text { State } 4 \text { respiration } \\
\text { (nmol/min/mg protein) }\end{array}$ & $10.5 \pm 1.4$ & $5.9 \pm 1.0^{*}$ & $11.8 \pm 1.7$ & $14.1 \pm 1.8$ \\
\hline ADP/O & $2.4 \pm 0.1$ & $2.3 \pm 0.03$ & $2.2 \pm 0.1$ & $2.1 \pm 0.04$ \\
\hline $\begin{array}{l}\text { Phosphorylation rate } \\
\text { (nmol ADP/min/mg } \\
\text { protein) }\end{array}$ & $298.7 \pm 44.6$ & $248 \pm 22.4$ & $278.1 \pm 8.3$ & $245.6 \pm 11.3^{*}$ \\
\hline
\end{tabular}

Heart mitochondria were isolated from rats fed a high caloric diet for 16 weeks versus controls on a standard laboratory chow. Respiration parameters were determined using glycolytic (glutamate) or fatty acid (malate and palmitoyl-L-carnitine [MPC]) as substrates. Values are expressed as mean \pm SEM ( $\mathrm{n} \geq 5$ animals). ${ }^{*} \mathrm{p}<0.05$ compared with age-matched controls. 


\section{Figure Legends}

Figure 1 Decreased cardiac contractile function in obese rats at baseline and in response to an ischemic insult. a) Baseline aortic output ( $\mathrm{ml} / \mathrm{min}$ ) for obese and control rats; b) Percentage aortic output recovery of rat hearts subjected to 15 minutes of global ischemia and reperfused for 10, 15 and 20 minutes, respectively; and c) Myocardial infarct size (expressed as \% of area at risk) after ischemia-reperfusion. The data are presented as mean \pm SEM. ${ }^{*} \mathrm{p}<0.05,{ }^{* *} \mathrm{p}<0.005$ vs. matched controls $(n=7-8)$.

Figure 2 Recovery of state 3 mitochondrial respiration in response to acute oxygen lack. Mitochondria from obese and control rats were isolated, supplied with $12.5 \mathrm{mM}$ malate and 54 $\mu \mathrm{M}$ palmitoyl-L-carnitine, and subjected to 20 minutes of oxygen lack followed by 6 minutes of reoxygenation. Recovery of respiratory function was evaluated by determining the ratio of postanoxia state 3 respiration over state 3 respiration prior to oxygen deficiency. The data are presented as mean \pm SEM. ${ }^{*} \mathrm{p}<0.05$ vs. controls $(\mathrm{n}=6)$. 
Figure 1A

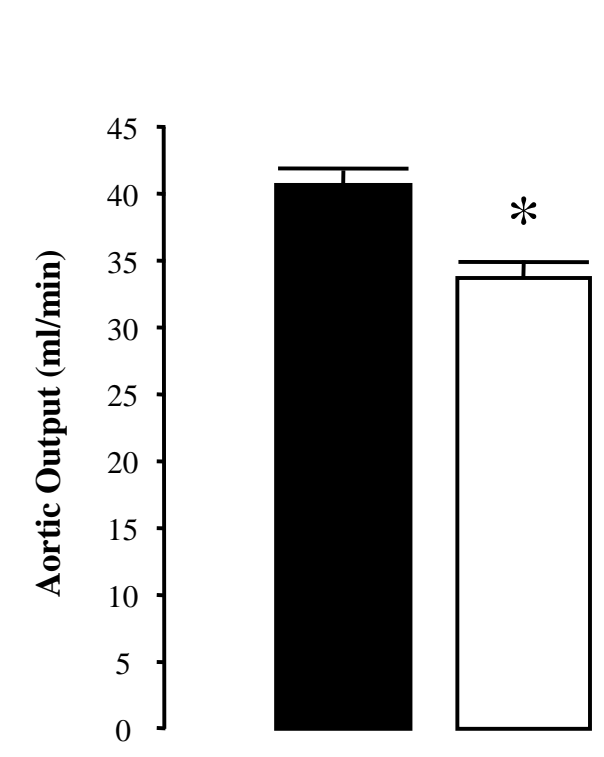

Figure 1B

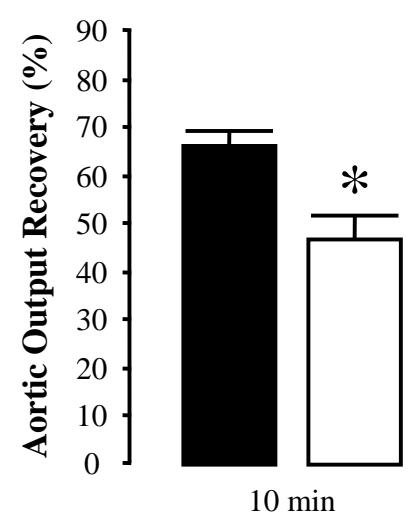

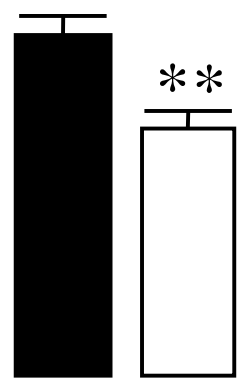

15 min
Control

$\square$ Obese 
Figure 1C

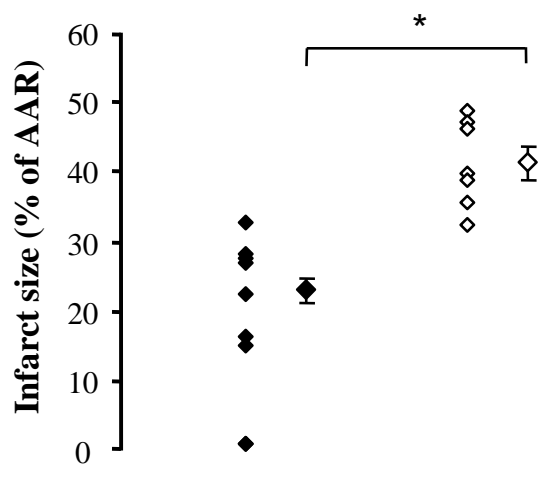

Figure 2

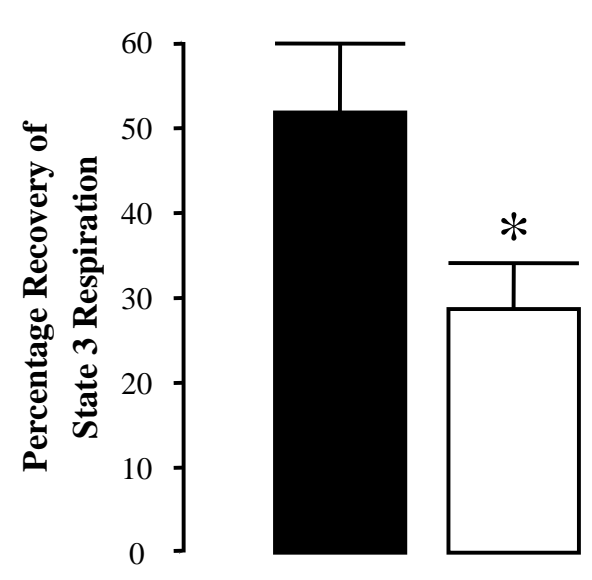

- Control

$\square$ Obese 